\title{
Diffusion-Dependent Mechanisms of Receptor Engagement and Viral Entry
}

\author{
Melissa M. Gibbons, ${ }^{\dagger}$ Tom Chou, ${ }^{\dagger}$ and Maria R. D'Orsogna*, \\ Department of Biomathematics, University of California, Los Angeles, Los Angeles, California, United States, \\ and Department of Mathematics, CalState-Northridge, Los Angeles, California, United States
}

Received: August 25, 2010

Enveloped viruses attach to host cells by binding to receptors on the cell surface. For many viruses, entry occurs via membrane fusion after a sufficient number of receptors have engaged ligand proteins on the virion. Under conditions where the cell surface receptor densities are low, recruitment of receptors may be limited by diffusion rather than by receptor-ligand interactions. We present a receptor-binding model that includes the effects of receptor availability at the viral binding site. The receptor binding and unbinding kinetics are coupled to receptor diffusion across the cell membrane. We find numerical solutions to our model and analyze the viral entry probabilities and the mean times to entry as functions of receptor concentration, receptor diffusivity, receptor binding stoichiometry, receptor detachment rates, and virus degradation/detachment rates. We also show how entry probabilities and times differ when receptors bind randomly or sequentially to the binding sites on the viral glycoprotein spikes. Our results provide general insight into the biophysical transport mechanisms that may arise in viral attachment and entry.

\section{Introduction}

Enveloped viruses gain entry into host cells by one of two mechanisms: endocytosis into the cell or fusion with the cellular envelope. ${ }^{1}$ In the case of endocytosis, viruses are engulfed by the cell membrane through a vesicle that is later degraded within the cell cytoplasm. The other dominant entry pathway of enveloped viruses, and the one in which we are interested in this study, is fusion between viral and host cell lipid membranes. Fusion is mediated by the interaction and binding of viral glycoprotein ligand spikes to cellular surface receptors. These interactions trigger conformational changes that lead to the formation of a pore between the two membranes, allowing for direct entry of the viral capsid into the cell. Many members of the Paramyxoviridae, Coronaviridae, and Retroviridae families of viruses fuse with host cells membranes, even at neutral $\mathrm{pH} .^{2}$

HIV-1 is among the most studied viruses entering cells via fusion. ${ }^{3}$ Upon contact with the cell, one of the many gp120/gp41 glycoprotein ligand spikes that coat HIV-1 binds to the cellular receptor $\mathrm{CD} 4$, causing a conformational change in the gp120 region of the spike. The binding site is now exposed and primed for the adsorption of coreceptors that are expressed on the cell surface, CCR5 or CXCR4, depending on cell type. These coreceptors activate additional conformational changes in the gp41 portion of the glycoprotein spike that finally lead to the fusion of the two membranes. During this process, multiple CD4, CCR5, and CXCR4 receptors and coreceptors may be engaged per glycoprotein spike, in stochiometries that have not been fully resolved and that may even vary from cell to cell. ${ }^{4-6}$ Other retroviruses that enter the cell by fusion include SIV, ${ }^{7}$ some subgroups of the murine leukemia virus, ${ }^{8}$ and the hepatatis B virus HBV. ${ }^{9}$

Most enveloped viruses utilize one or two ligand glycoprotein spikes to bind and fuse with the cellular plasma membrane. In certain cases, more may be required. For example, herpes simplex virus (HSV), a member of the Herpesviridae family, encodes about 12 different types of glycoproteins, of which four are known to be

* Corresponding author. Address for correspondence: Department of Mathematics, CSUN, Los Angeles, CA 91330-8313, USA. Tel.: (818) 6772703. E-mail: dorsogna@csun.edu.

$\uparrow$ University of California, Los Angeles.

* CalState-Northridge. engaged in the fusion process, ${ }^{10}$ each with their own associated receptor species. At times, infection by HSV can proceed even without any cellular receptor attachment. The Sendai and measles viruses, of the Paramyxoviridae family, utilize instead a single cell receptor species to gain entry into the cell, ganglioside receptors $(\mathrm{SA}-\mathrm{R})^{12,13}$ and CD46, ${ }^{14}$ respectively.

The above examples illustrate the many possibilities for virus - cell interactions and dynamics. Although specific details such as number and types of receptors may vary from system to system, the fundamental mechanism that leads to entry by fusion is the binding of viral ligands to cell receptors. ${ }^{15,16}$ Of course, ligand-receptor binding has been extensively studied both experimentally ${ }^{18,17}$ and theoretically ${ }^{19-22}$ over the past decades in many different chemical and biochemical contexts. More recently, however, kinetic models for ligand-receptor binding have been introduced to specifically address viral entry by fusion ${ }^{23,24}$ and binding of colloids to membranes. ${ }^{25}$ These models include the complex multistep binding reactions occurring at the cell membrane ${ }^{26}$ but mostly ignore the spatial variation and diffusion of receptors on the host cell surface, which may be important in certain cases. In particular, for the HIV-1 models mentioned above, ${ }^{23,24}$ it was assumed that an excess of cellular surface receptors was available for binding to HIV-1 glycoprotein ligand spikes so that their availability or diffusion to the contact area was not rate limiting.

The spatial arrangement of receptors, their mobility along the cell membrane, and their abundance on the cell may become limiting factors if the overall receptor concentration is low, or if the virus makes initial contact in a receptor-depleted region. Indeed, receptor and coreceptor levels in various families of cells have been measured, showing a wide variability not only across cell types but up to an order of magnitude density variations within the same cell species. ${ }^{27}$ Nonuniform distributions of receptors and coreceptors may be present even within a single cell. In particular, CD4, CCR5, and CXCR4 cluster to regions of the cell surface rich in the protein ezrin and where microvilli and other protrusions occur, ${ }^{28,29}$ leaving other areas mostly void. The CD4, CCR5, and CXCR4 receptors/coreceptors are not bound together and can be mostly treated as independent particles, although possible interplay between some species may arise. ${ }^{30}$ Other experiments showed that, while a 
subset of the CD4 population is immobile, the rest can freely diffuse on the surface, generally with diffusion constant comparable to that of the CCR5 coreceptors and of the order of $10^{-2} \mu \mathrm{m}^{2} / \mathrm{s} .^{31,30}$ In vitro studies have also revealed that HIV-1 viral loads are correlated with receptor and coreceptor availability. ${ }^{32,33}$ Pharmacologically, it has been found that reducing the surface concentration of CCR5 via specifically targeted drugs may hamper fusion between HIV-1 and T-cells ${ }^{34}$ and that treating T-cells with specific sphingomyelinase enzymes results in a reduced fusion probability of the HIV-1 virus due to restrictions in the surface mobility of surface CD4 receptors. ${ }^{35}$ Similarly, mouse melanoma cells engineered with reduced CD4 mobility resulted in a 7-fold reduction of HIV-1 fusion. ${ }^{36}$

The goal of this paper is to put the above experimental observations within a mathematical framework that can be adapted to model viral entry in general. We present and analyze a simplified kinetic model of receptor engagement and viral fusion that includes the effects of multiple receptor binding, degradation/detachment of the virus particle, and receptor diffusion. For simplicity, we will only consider a single receptor species and not distinguish between receptors and coreceptors. We will illustrate how each physical mechanism influences viral fusion, especially under conditions where receptor diffusion can be limiting. Our work is especially relevant for studying viral infectivity when total receptor or coreceptor concentrations are low, and for better understanding experiments that probe how receptor concentrations and mobility affect viral entry. Although our mathematical model is general, for concreteness, we will use model parameters consistent with data estimated from the virology literature. The basic model and its mathematical formulation are presented in the next section. In the Results and Discussion, we analyze numerical solutions of our model under various parameter regimes. Both probabilities of fusion and conditional mean times to fusion are studied as functions of receptor concentrations and diffusivity. We conclude by discussing possible refinements and extensions to our model.

\section{Materials and Methods}

The basic physical ingredients of our model include binding and unbinding of receptors, receptor diffusion, viral degradation, and irreversible membrane fusion. We first consider a virus particle initially nonspecifically adsorbed on a flat host cell surface via a glycoprotein ligand spike to which $N$ receptors must bind before the irreversible fusion process can take place (see Figure 1). We denote the number of bound receptors in the interim states by $n$, where $1 \leq n \leq N$. The binding of receptors is considered reversible for $1 \leq n<N$, while virus-cell membrane fusion that can occur when $n=N$ is irreversible. Receptors are freely diffusing on the cell membrane, so that the higher their abundance at the binding site, the higher the frequency of binding attempts. Mathematically, this assumption translates to the receptor-spike adsorption rate being dependent on the receptor concentration at the virus-cell contact area. In our model we do not include diffusion of the virus on the cell surface.

We assume cell receptors can be described via a radially symmetric, continuum field $c(r, t)$ diffusing on the cell surface with diffusivity $D$. At $t=0$ we allow the first virus - cell surface contact to be established at $r=0$ via nonspecific adsorption of a "naked" viral glycoprotein spike or aggregates of spikes whose typical imprint on the cell surface is a disk of radius $r=a$. We denote by $P_{n}(t)$ the probability of having $1 \leq n \leq N$ receptors attached and assume that the viral spike cannot accommodate more than $N$ receptors. Irreversible fusion can occur only from the final $N$-state when all receptors are bound to the glycoprotein

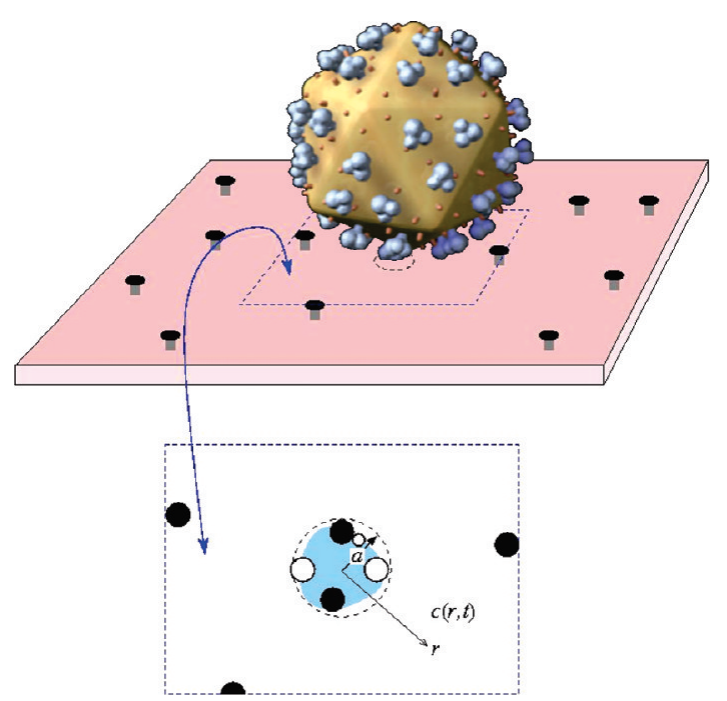

Figure 1. Schematic of the basic features of our model. The viral glycoprotein spike or aggregate of spikes centered about $r=0$ covers a footprint of radius $r=a$ on the flat cell membrane. The concentration of diffusing receptors is indicated by $c(r, t)$. In this figure, receptors are schematically depicted as dots. The glycoprotein spike has $N$ binding sites that must be fully occupied before the fusion process can occur. We allow the attachment of receptors to be fully reversible until the fusion state is reached, the viral contact spike to degrade, and receptors to freely diffuse on the cell surface.

spike. The initial condition is that, at time $t=0$, no receptors are bound to the viral spike protein, so that $P_{0}(t=0)=1$ and $P_{n}(t=0)=0$, for $n \neq 0$. Apart from receptor diffusion toward the viral spike complex, the virus is also subject to degradation due to external factors, such as proteasomes or digestion by other cellular enzymes. We investigate the conditions under which successful integration of the virus into the host cell may occur in competition with viral degradation.

The physical picture described above can be represented mathematically by studying the evolution of the probabilities $P_{n}(t)$ of having $n$ receptors adsorbed onto the viral glycoprotein spike at time $t$, and of the density $c(r, t)$ of diffusing receptors on the cell membrane. Our model consists of the following system of coupled differential equations:

$$
\begin{array}{r}
\frac{\mathrm{d} P_{0}(t)}{\mathrm{d} t}=-p_{0} c(r=a, t) P_{0}(t)+q_{1} P_{1}(t)-\mu_{0} P_{0}(t) \\
\frac{\mathrm{d} P_{n}(t)}{\mathrm{d} t}=-\left[p_{n} c(r=a, t)+q_{n}\right] P_{n}(t)+q_{n+1} P_{n+1}(t)+ \\
p_{n-1} c(r=a, t) P_{n-1}(t)-\mu_{n} P_{n}(t)
\end{array}
$$

$\frac{\mathrm{d} P_{N}(t)}{\mathrm{d} t}=p_{N-1} c(r=a, t) P_{N-1}(t)-q_{N} P_{N}(t)-\kappa_{\text {in }} P_{N}(t)-\mu_{N} P_{N}(t)$

$$
\begin{gathered}
\frac{\mathrm{d} P_{\text {in }}(t)}{\mathrm{d} t}=\kappa_{\text {in }} P_{N}(t) \\
\frac{\partial c(r, t)}{\partial t}=D\left(\frac{\partial^{2}}{\partial r^{2}}+\frac{1}{r} \frac{\partial}{\partial r}\right) c(r, t)
\end{gathered}
$$

Here $\kappa_{\text {in }}$ is the rate of fusion into the cell when $N$ receptors are bound to the viral spike, $D$ is the receptor diffusion coefficient 
on the cell membrane, and $\mu_{n}$ is the viral degradation rate, which can vary as a function of the number $n$ of bound receptors. The last equation describes the diffusion of a circularly symmetric receptor concentration field on a flat two-dimensional cell surface.

Receptor binding and unbinding rates for states where $n$ receptors are already attached to the spike are denoted by $p_{n} c(r=a, t)$ and $q_{n}$, respectively. In our notation, we have explicitly assumed that the attachment rate $p_{n} c(r=a, t)$ is linearly proportional to the number of receptors at the contact perimeter $r=a$. The specific functional forms of $p_{n}$ and $q_{n}$ will depend on the details of the receptor binding process. For example, highly cooperative receptor binding $p_{n}$ could be modeled by a nonlinearly increasing function of $n$. In this paper, we consider the two complementary cases of random or sequential adsorption. Random binding implies that an incoming receptor can attach to any of the unoccupied sites that are available on the viral spike, while all bound receptors are equally likely to detach. Sequential receptor binding arises when the binding sites are molecularly distinguishable so that receptor binding and unbinding follow a specific order and receptors can attach or detach only to or from certain predetermined sites. We represent these two binding mechanisms by their distinct functional forms of the effective binding and unbinding rate $p_{n} c(r=a, t)$ and $q_{n}$ :

$$
\begin{gathered}
p_{n} c(r=a, t)=(N-n)^{\alpha} p c(r=a, t) \\
q_{n}=n^{\alpha} q
\end{gathered}
$$

Here, $p$ and $q$ represent the intrinsic kinetic rates between a cell receptor and a binding site of the virus glycoprotein spike. The index $\alpha=1$ corresponds to the random receptor binding/unbinding mechanism where the effective binding rate $p_{n}$ is proportional to the total number of ways $N-n$ an unoccupied receptor site can be filled and, as mentioned earlier, to the free receptor concentration $c(r=a, t)$ at the contact region. Similarly, the effective detachment rate $q_{n}$ is proportional to the number of bound receptors $n$. The case $\alpha$ $=0$ corresponds to the sequential binding/unbinding mechanism. Here, incoming receptors can bind to only one specific site regardless of $n<N$. We also assume that, at each stage $n>0$, only one specific receptor can detach, regardless of $n$, so that $q_{n}=q$.

Similarly to the attachment and detachment rates, many scenarios arise for the modeling choices of the degradation rates $\mu_{n}$. One possible assumption is that the cellular degradation factors embodied in $\mu_{n}$ act only when the virus has no receptors attached, so that $\mu_{0}>0$ and $\mu_{n}=0$, for all $n>0$. Alternatively, we can assume the cascade of degradation rates is uniform over the range of intermediate states $n$ and $\mu_{n}$ is a constant, independent of $n$.

The initial receptor concentration is assumed to be uniformly distributed so that $c(r, t=0)=c_{\infty}$. We also impose the boundary condition $c(r \longrightarrow \infty, t)=c_{\infty}$, far away from the viral contact region at $r=a$. The model is completed by the boundary condition at the viral spike-host cell contact perimeter at $r=a$, as shown in Figure 1.

$$
-\sum_{j=1}^{N} q_{j} P_{j}(t)+\sum_{j=0}^{N-1} p_{j} c(r=a, t) P_{j}(t)=2 \pi a D \hat{r} \cdot \nabla c(r=a, t)
$$

TABLE 1: Physical Parameters of HIV-1 That Will Be Used To Guide This Study

\begin{tabular}{lll}
\hline \multicolumn{1}{c}{ parameter } & \multicolumn{1}{c}{ HIV-1 } & refs \\
\hline receptor diffusion, $D$ & $0.044 \mu \mathrm{m}^{2} / \mathrm{s}$ & {$[35]$} \\
receptor concentration, $c_{\infty}$ & $300-3000 \mathrm{CD} 4 / \mu \mathrm{m}^{2}$ & {$[32]$} \\
receptor binding, $p$ & $\approx 1 \mathrm{~s}^{-1}$ & {$[41]$} \\
receptor unbinding, $q$ & $\approx 4 \mathrm{~s}^{-1}$ & {$[41]$} \\
viral degradation, $\mu_{0}$ & $\approx 15 \mathrm{~s}^{-1}$ & {$[37]$} \\
viral imprint radius, $a$ & $\approx 0.01 \mu \mathrm{m}$ & {$[38]$}
\end{tabular}

Equation 8 balances the incoming receptor flux from the cell surface into the virus - cell contact area of radius $r=a$ on the RHS, with the material that is attaching to and detaching from the viral spike on the LHS. The quantities we are interested in evaluating are the total time integrated probability $P_{\text {in }}$ that the virus will enter the cell, and the mean first time to entry, $T$. These are calculated from the following integrals:

$$
\begin{gathered}
P_{\text {in }}=\kappa_{\text {in }} \int_{0}^{\infty} P_{N}(t) \mathrm{d} t \\
T=\kappa_{\text {in }} P_{\text {in }}^{-1} \int_{0}^{\infty} t P_{N}(t) \mathrm{d} t
\end{gathered}
$$

where the normalization factor $P_{\text {in }}{ }^{-1}$ included in the mean entry time $T$ is used to condition the virus on not having degraded before entering the cell.

2.1. Diffusion-Limited Receptor Engagement. The system of eqs $1-5$ can be used to study a variety of microbiological systems where viruses or other external compounds have made an initial contact with a cell, but where the diffusion of surface proteins to the contact point is necessary before fusion can be triggered. For concreteness, we focus on the viral spike-cellular receptor system described earlier and perform simulations using a range of physical parameters estimated from the experimental virology literature, ${ }^{37}$ particularly for HIV-1. These are compiled in Table 1. Since not all physical parameters are experimentally accessible, where necessary we use plausible estimates, as indicated in the text.

The nonlinear coupling between cell surface receptor concentration and the occupation probabilities of the virus spike, $c(r, t) P_{n}(t)$, requires the use of numerical methods to solve for the probabilities $P_{n}$ from which the quantities of interest eqs 9 and 10 can be derived. The receptor concentration field is assumed to be circularly symmetric about the disk of viral contact, so we perform spatial discretization only in the radial direction. In all of our numerical results, the radius of the viral contact area is set to be $a=0.01 \mu \mathrm{m}$, which is consistent with the size of many viruses. ${ }^{38}$ The maximum radius for computational purposes is set to be $r_{\max }=1 \mu \mathrm{m}$. We choose to discretize our surface into 100 equally spaced points, so that at $r=r_{\max }$, the boundary condition on the receptor concentration $c\left(r=r_{\max }\right)$ $=c_{\infty}$ is enforced. The Laplacian is computed using the central finite difference formula, ${ }^{39}$ and a fourth-order Runge-Kutta scheme, which is an explicit forward-stepping method, is used for time stepping. ${ }^{40}$

2.2. Nondimensionalization. In this section, we nondimensionalize eqs $1-5$ and the boundary conditions by measuring length in units of $a$, the radius of the virus spike-cell membrane contact area, and time in units of $a^{2} / p$. Estimates of $a \approx 0.01$ $\mu \mathrm{m}$ are readily available in the literature. ${ }^{38}$ On the other hand, experimental measurements of the kinetic binding rate $p$ are extremely difficult to obtain. However, an estimate of $p \approx 1$ $\mu \mathrm{m}^{2} / \mathrm{s}$ is consistent with qualitative observations in one single- 
molecule study. ${ }^{41}$ Upon introducing the renormalized length and time scales, the dimensionless counterparts to all parameters are given as

$$
\begin{gathered}
\bar{r}=\frac{r}{a}, \quad \bar{t}=\frac{t p}{a^{2}}, \quad \bar{c}(\bar{r}, \bar{t})=a^{2} c(r, t) \\
\bar{D}=\frac{D}{p}, \quad \bar{q}=\frac{q a^{2}}{p}, \quad \bar{\kappa}_{\mathrm{in}}=\frac{\kappa_{\mathrm{in}} a^{2}}{p}, \quad \bar{\mu}_{n}=\frac{\mu_{n} a^{2}}{p}
\end{gathered}
$$

The above choice of nondimensionalization leads to the position of the viral spike-cell contact boundary being located at $\bar{r}=$ 1. Equations $1-5$ remain in exactly the same form, with all parameters replaced by the nondimensional quantities listed above. The dimensionless boundary condition derived from eq 8 is

$$
-\sum_{j=1}^{N} \bar{q}_{j} P_{j}(\bar{t})+\sum_{j=0}^{N-1} \bar{p}_{j} \bar{c}(\bar{r}=1, \bar{t}) P_{j}(\bar{t})=2 \pi \bar{D} \hat{\bar{r}} \cdot \nabla \bar{c}(\bar{r}=1, \bar{t})
$$

where the nondimensional binding and unbinding rates are

$$
\begin{gathered}
\bar{p}_{n} \bar{c}(\bar{r}=1, \bar{t})=(N-n)^{\alpha} \bar{c}(\bar{r}=1, \bar{t}) \\
\bar{q}_{n}=n^{\alpha} \bar{q}
\end{gathered}
$$

With these nondimensionalization choices, our spatial discretization runs from $\bar{r}=1$ to $\bar{r}=100$, and all quantities listed in Table 1 are rescaled according to eqs 11. In the following sections, nondimensional values for all parameters will be used. For notational simplicity, we will suppress the overbar notation.

\section{Results and Discussion}

3.1. Random Receptor Binding. In this section, we study the case of random receptor binding, where $\alpha=1$. We first assume that cellular degradation acts only on the "naked" glycoprotein spike so that the nondimensional degradation rates $\mu_{0}>0$ and $\mu_{n}=0$ for $n \neq 0$. Figure 2 shows the probability $P_{\text {in }}$ of the virus penetrating the cell as a function of the nondimensional receptor concentration $c_{\infty}$, degradation rate $\mu_{0}$, and receptor diffusion coefficient $D$, when the number of receptors necessary for entry is $N=4$ (left) and $N=8$ (right). Nondimensional kinetic rates are set to $\kappa_{\text {in }}=q=0.0001$.

As shown in Figure 2, when $\mu_{0}=0$, the time-integrated entry probability $P_{\text {in }}=1$ for all positive receptor diffusion coefficients and receptor concentrations. The fact that $P_{\text {in }}$ is a decreasing function of $\mu_{0}$ is expected since only degradation represented by $\mu_{0}>0$ can diminish the total entry probability. Even in the presence of degradation $\left(\mu_{0}>0\right)$, the fusion probability $P_{\text {in }} \rightarrow$ 1 provided the receptor concentration $c_{\infty} \rightarrow \infty$. Figure 2 also confirms that $P_{\text {in }}$ is an increasing function of the receptor diffusion coefficient $D$. Indeed, increasing $D$ replenishes the concentration of receptors near the glycoprotein spike, speeding the receptor engagement process and increasing the probability of fusion. These qualitative results reveal that as long as $c(r=$ $1, t)$ is sufficiently large, either due to large $D$ or large $c_{\infty}, P_{\text {in }}$ will be near unity.

For the range of the parameters $c_{\infty}, \mu_{0}$, and $D$ shown in Figure 2 , the entry probabilities remain relatively insensitive to changes
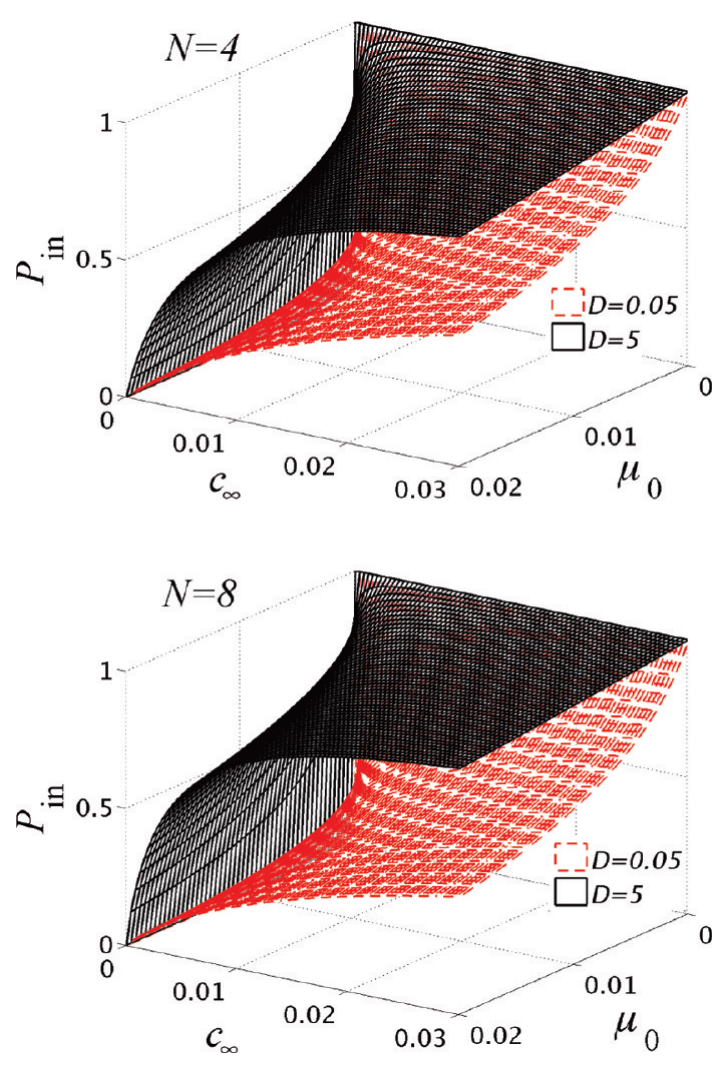

Figure 2. Probability of viral entry as a function of receptor concentration $c_{\infty}$, and viral degradation $\mu_{0}$, for various $D$, when the number of receptors needed for fusion is $N=4$ (left) and $N=8$ (right). Here, we assume random receptor binding and let degradation act only at the $n=0$ state, with magnitude $\mu_{0}$. Other parameters used are $\kappa_{\text {in }}=$ $q=0.0001$. All quantities are dimensionless.

in the number of receptors $N$ necessary for fusion when $N$ goes from $N=4$ to $N=8$. However, there is an interesting quantitative transition in $P_{\text {in }}$. As shown in Figure 3, at higher diffusion coefficients (e.g., $D=5$ ), the entry probability is higher when $N=8$ than when $N=4$. As $D$ is lowered, there is switching between the $N=4$ and $N=8$ surfaces so that for lower $D$ values, the $N=4$ case results in a higher entry probability $P_{\text {in. }}$. This transition can be understood as follows.

For random receptor binding, the effective attachment rate $p_{0} c(r=1, t)=N c(r=1, t)$ from the naked $n=0$ state toward the first occupied one at $n=1$ is twice as large for $N=8$ than for $N=4$. On the other hand, the unbinding rates $q_{n}=q n$ are independent of $N$. When the diffusion coefficient is large, the $c(r=1, t) \lesssim c_{\infty}$ term is effectively constant; hence, a system with larger $N$ escapes degradation by leaving the $n=0$ unbound state faster, resulting in a higher entry probability $P_{\text {in }}$. For smaller diffusion constants, $c(r=1, t) \ll c_{\infty}$ due to depletion, and the increase of the binding rate with increasing $N$ is counteracted by decreases in the local receptor concentration.

In Figure 4, we plot the conditional mean time to viral entry, $T$ (using eq 10), as a function of receptor concentration $c_{\infty}$ and degradation rate $\mu_{0}$. We used the same parameters values as those used to generate Figure 2. Mean entry times are rather insensitive to receptor diffusivity $D$ except at very low receptor concentrations $c_{\infty}$ where receptor binding is highly diffusionlimited. In fact, throughout our analyses, the dependence on receptor diffusivity $D$ vanishes when $c_{\infty} \rightarrow \infty$ since the kinetics are in no way limited by transport to the glycoprotein binding sites. However, the conditional mean entry time $T$ is a decreasing function of $\mu_{0}$ and $c_{\infty}$. As degradation expressed via $\mu_{0}$ increases, viral entry can occur only if viruses bind receptors quickly, 

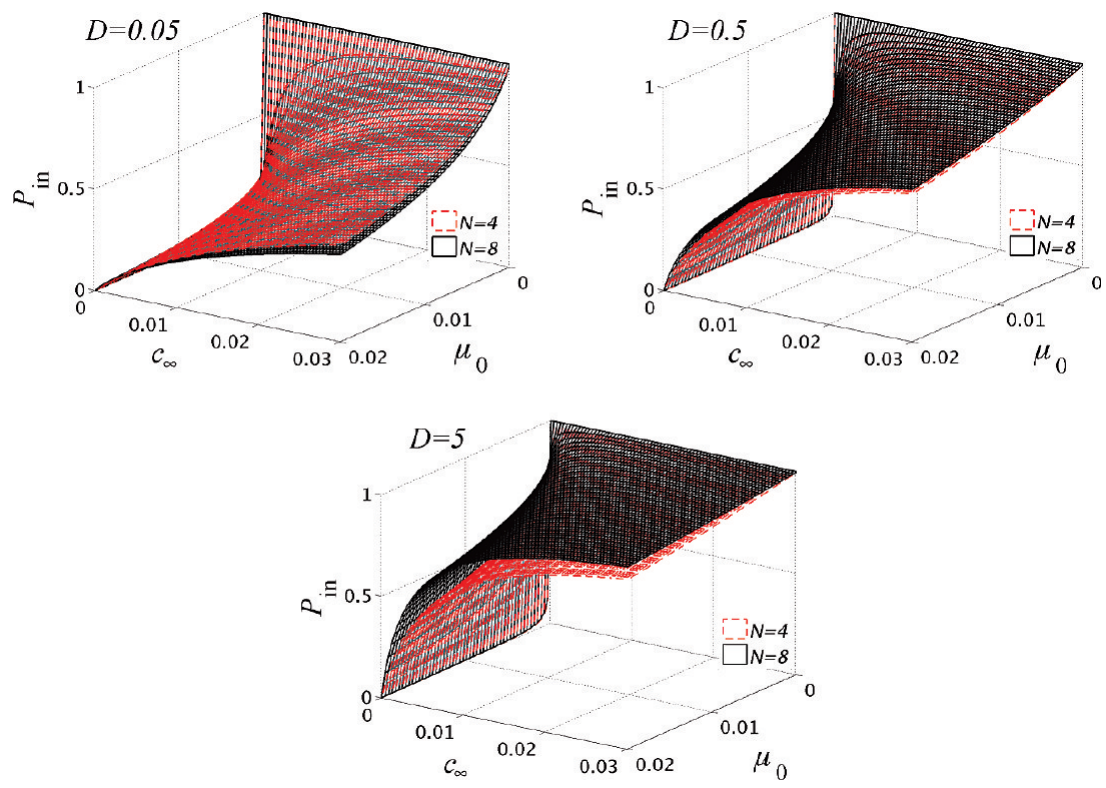

Figure 3. Probability of entry is higher for larger $N$ as the diffusion coefficient is increased. All quantities are dimensionless.
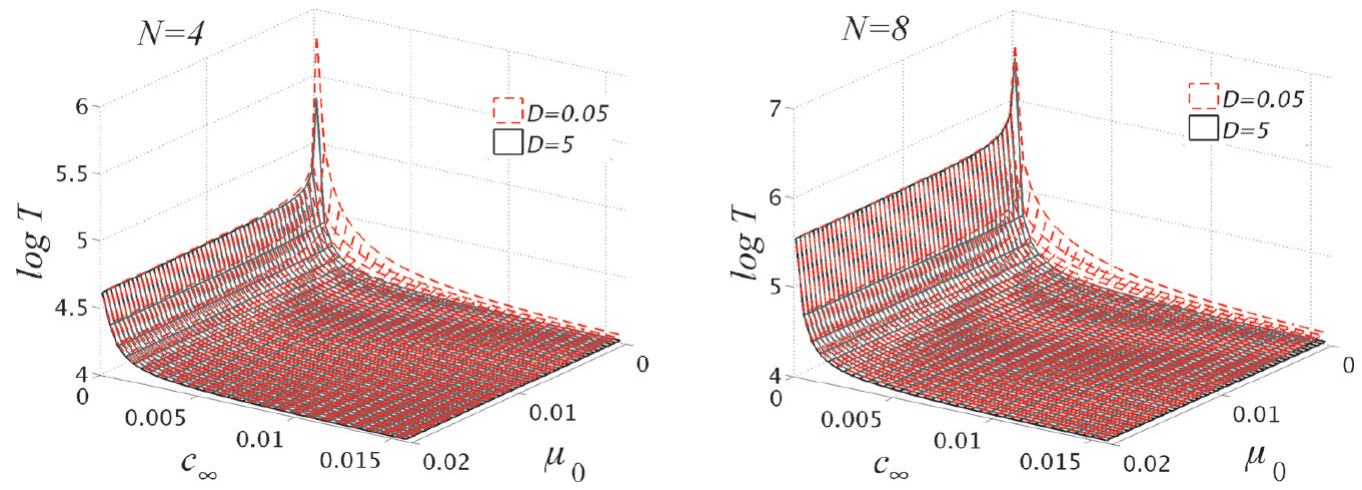

Figure 4. Average time to viral entry $T$ as a function of receptor concentration $c_{\infty}$, and viral degradation/desorption $\mu_{0}$, when the number of receptors needed for fusion is $N=4$ (left) and $N=8$ (right). Each plot overlays three surfaces corresponding to receptor diffusivities $D=0.05$, 5. We assume random receptor binding and that degradation occurs only at $n=0$. As $c_{\infty}$ and $\mu_{0}$ are increased, in both cases of $N=4$ and $N=8$, $T$ tends to saturate at a finite value. All quantities are dimensionless.

leading to a smaller conditional entry time $T$. Similarly, for all values of $\mu_{0}$, increases in $c_{\infty}$ enable faster receptor engagement, decreasing $T$. For very large $\mu_{0}$ (not plotted) the entry time $T$ asymptotes to a finite value because only the initial state $n=0$ is subject to degradation. Even though viral entry requires fast binding of the first receptor in order to escape fast degradation at $n=0$, the binding of the remaining $N-1$ receptors and subsequent fusion can occur over a longer amount of time. Our main finding is that, even though entry probabilities vary significantly across the parameter regimes studied, the conditional mean entry time is relatively insensitive to $D, \mu_{0}$, and $c_{\infty}$, except at very low receptor concentrations.

We now consider the scenario where degradation can occur at all levels $n$ of receptor binding, implemented via a constant degradation rate $\mu_{n}=\mu_{0} /(N+1)$. Figure 5 shows $P_{\text {in }}$ as a function of receptor concentration $c_{\infty}$, and intrinsic viral degradation rate $\mu_{0}$, when the number of receptors needed for fusion is $N=4$ (left) and $N=8$ (right). As expected, the entry probabilities are higher for larger $D$, especially when $c_{\infty}$ is small. Since increasing the diffusion coefficient does not significantly increase the entry probability $P_{\text {in }}$, the entry behavior in this regime is dominated by the degradation term. However, compared to the case where degradation occurs only at the receptor-free state ( $\mu_{n}=0$ for $n \neq 0$, as shown in Figure 2), uniform degradation dramatically decreases the entry prob- ability as $\mu_{n}=\mu_{0} /(N+1)$ increases. Even though the relative total strength of degradation is the same as that in Figure 2, the virus can now be degraded at any state $n$ before the final irreversible fusion process, and it is not enough to escape the $n=0$ state to avoid degradation. $P_{\text {in }}$ is thus lower in Figure 5 than in Figure 2.

In Figure 6, we plot the conditional mean time to entry $T$ for the uniform degradation case where $\mu_{n}=\mu_{0} /(N+1)$ as a function of $\mu_{0}$ and $c_{\infty}$. As described above, in this case, degradation is not confined to the $n=0$ case and is present for all $n$ values, resulting in an overall higher degradation probability (except when $\mu_{0}=0$ ) during the set of attachment/detachment events leading to full occupancy $N$. This higher degradation likelihood translates to shorter mean entry times for the uniform degradation case compared to the $\mu_{n}=0$ for $n \neq 0$ case, because of the conditional nature of $T$ in eq 10. Since degradation is present at all $N$ stages, the entire entry process must be accelerated for fusion to occur.

In contrast to the conditional mean entry time shown in Figure 4 , where $T$ asymptotes to a finite value in the large $\mu_{0} \rightarrow \infty$ limit, in Figure $6, T \rightarrow 0$ in the large $\mu_{0}$ limit. If $\mu_{n}=0$ for $n$ $\neq 0$, competition between entry and degradation occurs only at $n=0$, and tuning $\mu_{0}$ will only affect the time to go through the $n=0$ state. The higher $\mu_{0}$, the quicker the time to enter the $n$ $=1$ state, due to the conditional nature of the entry process. 

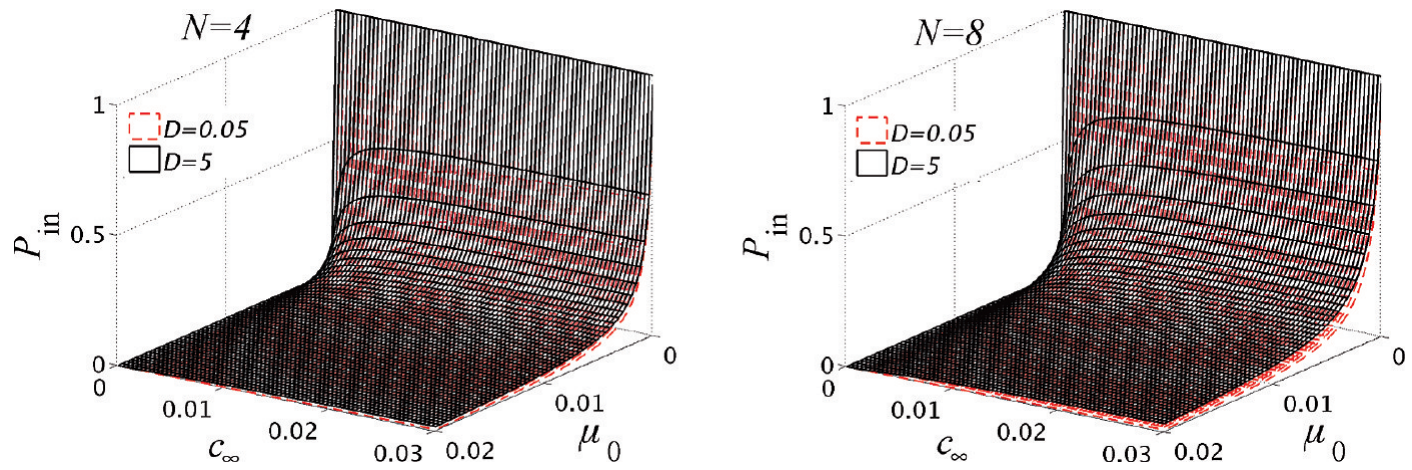

Figure 5. Probability of viral entry as a function of receptor concentration $c_{\infty}$, and viral degradation $\mu_{0}$, for various $D$, when the number of receptors needed for fusion is $N=4$ (left) and $N=8$ (right). We assume random receptor binding and allow degradation to apply uniformly to all $n$ states so that $\mu_{n}=\mu_{0} /(N+1)$. In this plot we have used $\kappa_{\text {in }}=q=0.0001$. All quantities are dimensionless.
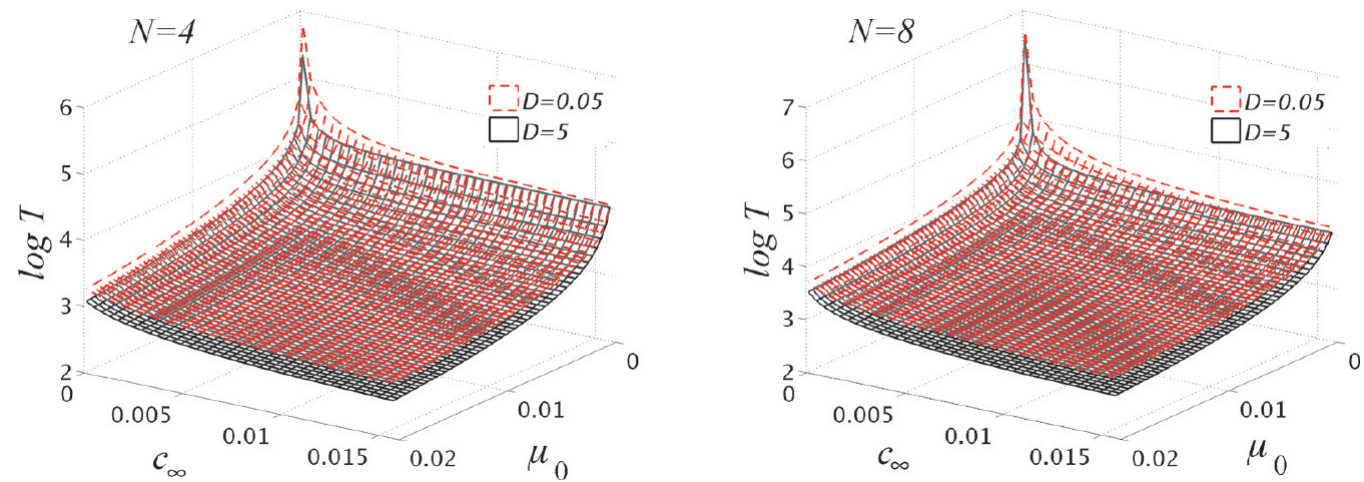

Figure 6. Average time to viral entry as a function of receptor receptor concentration $c_{\infty}$, and viral degradation $\mu_{0}$, for various values of $D$, when the number of receptors needed for fusion is $N=4$ (left) and $N=8$ (right). We assume random receptor binding and allow degradation to apply uniformly to all $n$ states so that $\mu_{n}=\mu_{0} /(N+1)$. In this plot we have used $\kappa_{\text {in }}=q=0.0001$. All quantities are dimensionless.
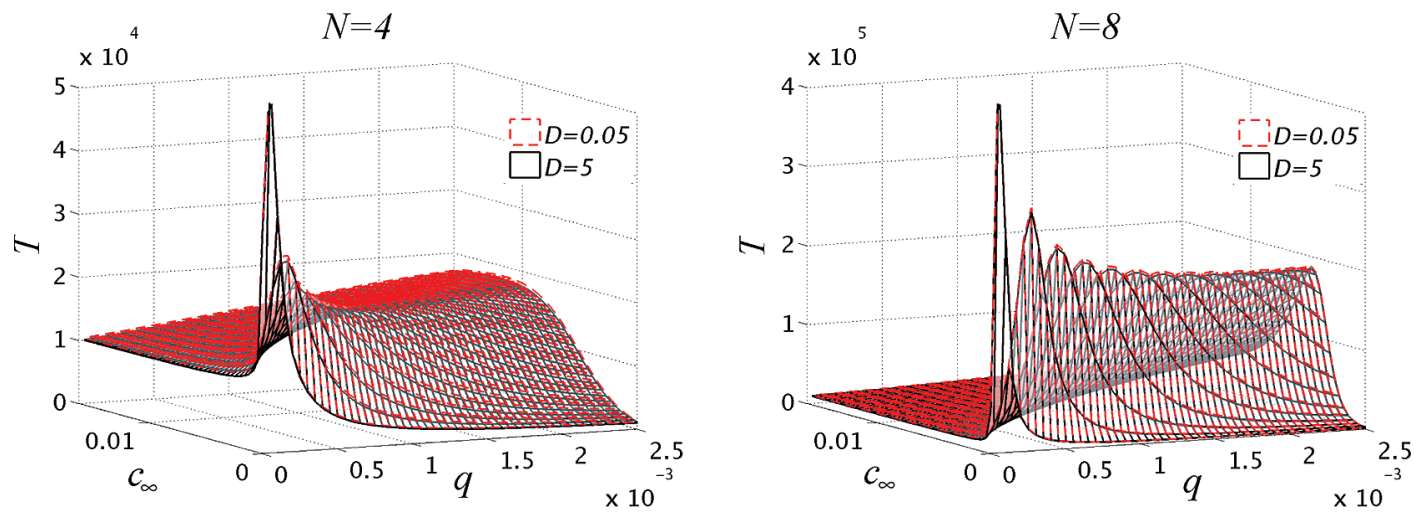

Figure 7. Conditional mean time to viral entry as a function of receptor concentration $c_{\infty}$, and receptor unbinding rate $q$, for $N=4$ (left) and $N$ $=8$ (right) and $D=0.05,5$. We set $\kappa_{\text {in }}=0.0001$, assume random receptor binding, and allow degradation to occur only at $n=0$ with rate $\mu_{0}=$ 0.015. While different axis values are used for mean entry time, the value for $N=4$ and $N=8$ at larger receptor concentrations is in fact the same, showing that the main contribution to $T$ is given by the time to exit the $n=0$ state. All quantities are dimensionless.

Since degradation does not affect the other $n \neq 0$ states, the time to traverse them will not change upon changing $\mu_{0}$. As a result, for large $\mu_{0}$, the first time to infection is dominated by the time to reach $N$ starting from $n=1$ and bears very little dependence on the minuscule time to exit the $n=0$ state, the only state where degradation arises. Once the time to exit the state $n=0$ is low enough, $T$ will asymptote to a finite value as $\mu_{0}$ is increased, since it is passage through the other states that determines the mean first passage dynamics. On the other hand, when degradation is possible at each step, increasing the degradation rate $\mu_{0}$ requires that transitions across all $n$ be faster in order to escape annihilation. Hence, increasing $\mu_{0}$ will continuously decrease the mean first entry time $T$ to zero.

We can also study how the relative magnitude of the kinetic rate constants affects the viral entry probabilities. Upon varying the unbinding rate constant $q$, and letting degradation act only on the $n=0$ unbound state, the probabilities $P_{\text {in }}$ are monotonic in $D$ and $c_{\infty}$ and quite similar to those shown in Figure 2 for each value of $q$. However, nonmonotonic behavior emerges in the dependence of the conditional mean entry time $T$ on $c_{\infty}$ and $q$, as shown in Figure 7 for $N=4$ (left) and $N=8$ (right). For both $N$ values, and a fixed value of receptor detachment $q$, there exists a peak in the conditional mean entry time $T$ as a function of $c_{\infty}$. This may be explained as follows. At high receptor concentrations $c_{\infty}, T$ decreases with increasing $c_{\infty}$, since there is a higher availability of receptors. Furthermore, the only state where degradation may occur, $n=0$, is rather unlikely to be heavily populated as long as $c_{\infty} \gtrsim q$ so that the effective binding rate $p_{0} c(r=1, t)$ is larger than the unbinding rate $q$. However, as the nondimensional receptor concentration is reduced to $c_{\infty}$ 

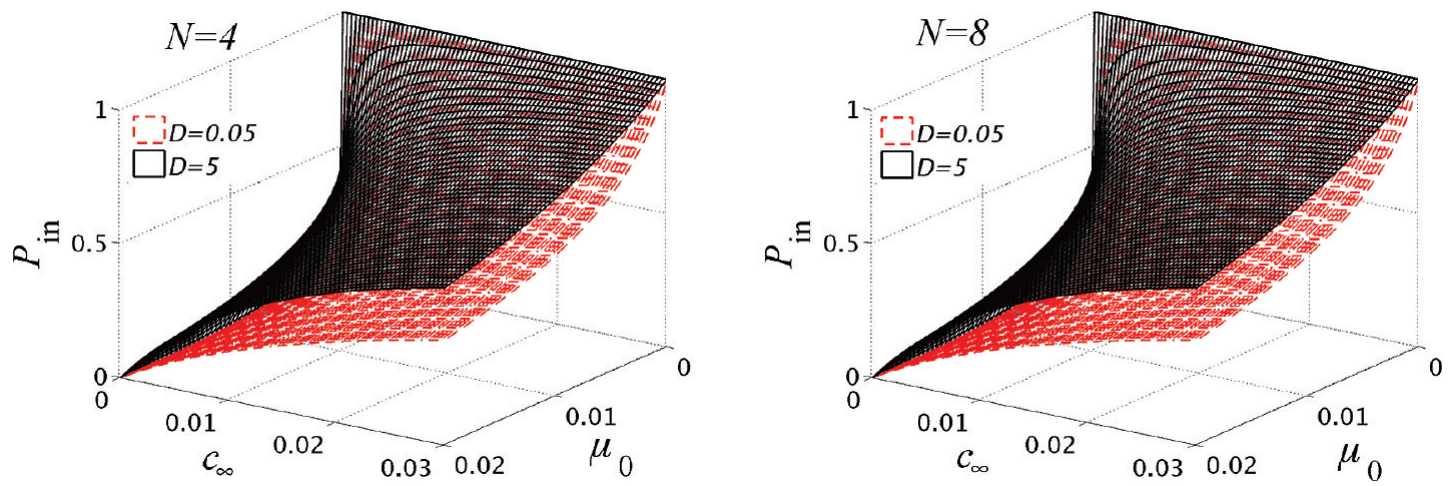

Figure 8. Probability of viral entry as a function of receptor concentration $c_{\infty}$ and viral degradation $\mu_{0}$, for $N=4$ (left) and $N=8$ (right) at values of $D=0.05,5$. We assume sequential receptor binding and allow degradation to occur only at $n=0$. The other parameters used are $\kappa_{\text {in }}=q=$ 0.0001. All quantities are dimensionless.
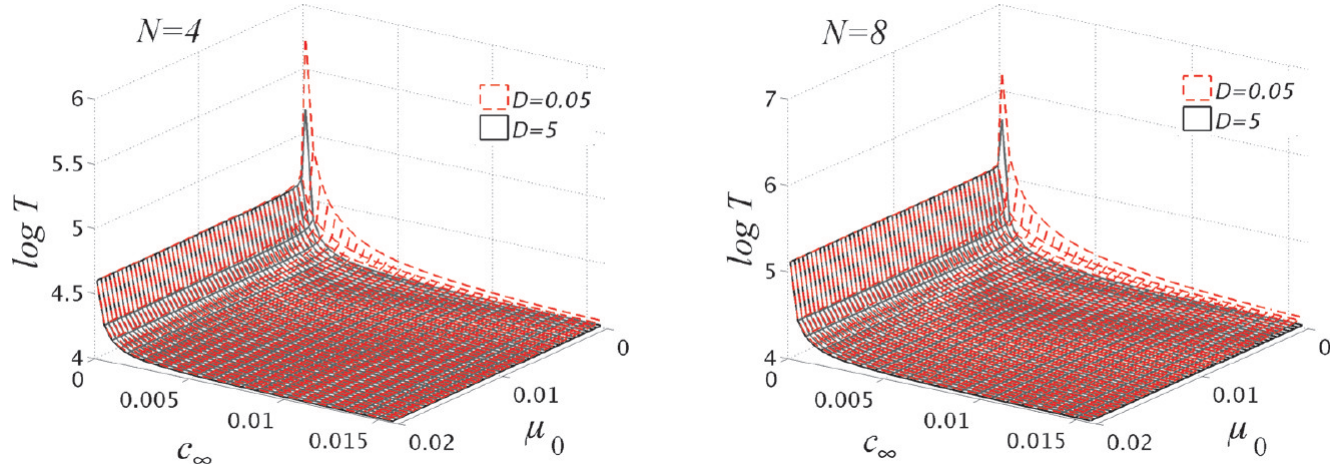

Figure 9. Average time to viral entry as a function of receptor concentration $c_{\infty}$ and viral degradation $\mu_{0}$, for $N=4$ (left) and $N=8$ (right) and various receptor diffusivities $D$. We assume sequential receptor binding and that degradation acts only at $n=0$. All quantities are dimensionless.

$\simeq q$, the $n=0$ state becomes more likely to be occupied, and degradation becomes more likely to occur. The conditional nature of the entry time $T$ now plays its role so that viruses that do enter the cell must do so quickly in order to avoid being degraded. The lower $c_{\infty} \simeq q$ is, the more this effect is pronounced, the faster the entry time $T$. These two trends, decreasing $T$ with decreasing $c_{\infty}$ for $c_{\infty} \lesssim q$ and decreasing $T$ with increasing $c_{\infty}$ for $c_{\infty} \gtrsim q$, give rise to the peak seen in Figure 7. Its maximum is located at approximately $c_{\infty} \simeq q$ and becomes more pronounced as $N$ increases. We do not vary $\kappa_{\text {in }}$ in this work, since changes in the fusion rate do not qualitatively affect mean first passage times nor entry probabilities.

3.2. Sequential Receptor Binding. In this section, we consider viral entry dynamics for the $\alpha=0$ case of sequential adsorption using the same parameter values used in the previous sections and in Figures 2 and 4. Here, we only consider the case when degradation acts only on the $n=0$ state, with magnitude $\mu_{0}$. We do not consider the case of uniformly spread degradation, where $\mu_{n}=\mu_{0} /(N+1)$, since first passage times and probability entries are qualitatively similar to those shown here for the nonuniform degradation case where $\mu_{n}=0$ for $n$ $\neq 0$.

Upon plotting $P_{\text {in }}$ as a function of $\mu_{0}$ and $c_{\infty}$ in Figure 8 , we see that all qualitative features of the surface plots are similar to the case of random receptor binding. However, for a given set of physical parameters, the entry probability is lower in the present case. Since binding rates $p_{n}$ are proportional to $(N-$ $n)^{\alpha}$, attachment rates are higher for random binding at $\alpha=1$ than for sequential binding at $\alpha=0$. Viral spikes on which receptors attach randomly are thus less likely to remain in the $n=0$ state and less likely to be subject to degradation. Thus, the cumulative viral entry probability $P_{\text {in }}$ is higher in the random adsorption case than in the sequential binding one. Moreover, since $p_{n}$ and $q_{n}$ are not functions of $N$ when sequential binding is assumed, the entry probability does not go through the transition at large diffusion coefficients as seen in Figure 3. In fact, there is not even a quantitative difference in the entry probability as $N$ is increased from $N=4$ to $N=8$. This is a consequence of assuming that the degradation rate $\mu_{0}$ only acts when zero receptors are bound, and that this is the only factor that reduces the entry probability. Indeed, in the sequential case, whether $N=4$ or $N=8$, having identical binding and unbinding rates imply that while the system with $N=8$ may take a longer time to enter the cell, especially at low $c_{\infty}$, the total entry probability is the same, resulting in identical values of $P_{\text {in }}$.

The conditional mean entry time for sequential receptor binding is plotted in Figure 9 as a function of $c_{\infty}$ and $\mu_{0}$, and shows a quantitative dependence on $N$. As with random receptor binding, $T$ only varies significantly at low receptor concentration values. For low $c_{\infty}$, it takes the virus about three times as long to enter the cell when $N=8$ than when $N=4$. At moderate concentrations $c_{\infty}$ for all degradation values used, the average time to entry shows little dependence on $N$ or $D$. These results are very similar to those illustrated in the previous section when random receptor binding was used (cf. Figure 4). Overall, the $N=4$ surfaces for $T$ are quantitatively similar regardless of the binding mechanism, while the $N=8$ surfaces show that, at low $c_{\infty}, T$ is larger when random receptor binding is assumed. This larger $T$ arises because at low $c_{\infty}$, the random unbinding rates $q_{n}=n q$ are overwhelmingly larger than the binding rates $(N-n) c(r=1, t)$, increasing the time spent at low $n$ values, compared to the sequential case where $q_{n}=q$.

However, the differences between random and sequential binding, and between $N=4$ and $N=8$, are minor and occur for values of $c_{\infty}$ that would physically be considered very low. ${ }^{32,41}$ For moderate, physical values of receptor concentrations 

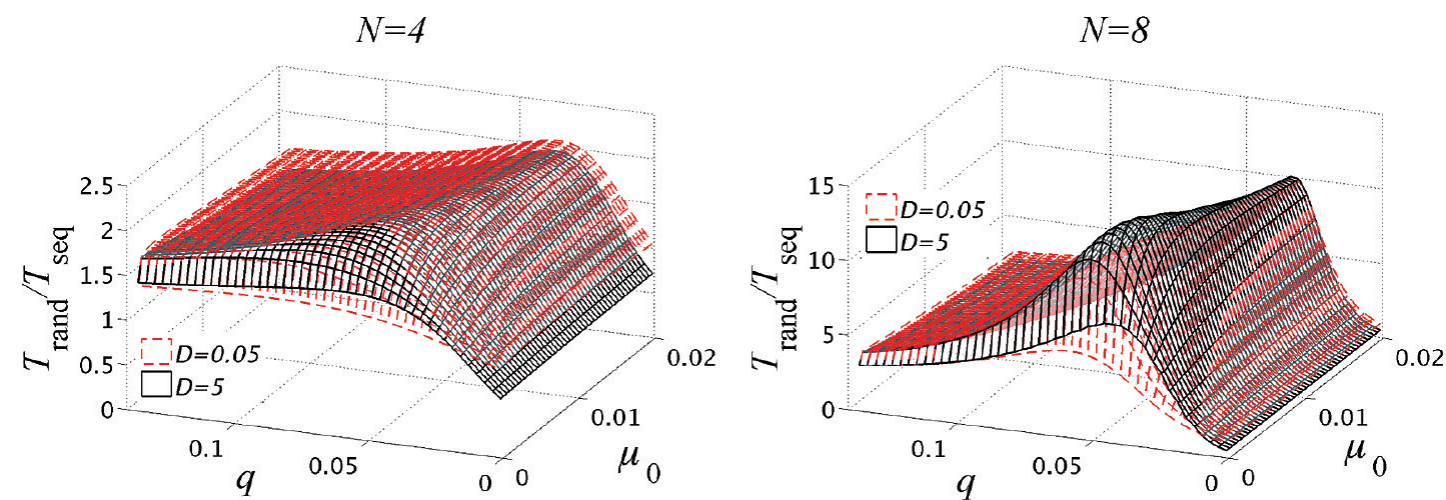

Figure 10. Ratio $T_{\text {rand }} / T_{\text {seq }}$ of average time to viral entry as a function of receptor unbinding rate $q$, degradation $\mu_{0}$, and diffusion coefficient $D$, when the number of receptors needed for fusion is $N=4$ (left) and $N=8$ (right). Here, $c_{\infty}=\kappa_{\text {in }}=0.03$ and degradation/desorption is assumed to act only at $n=0$. All quantities are dimensionless.

and degradation, the average time to entry is relatively constant across a large range of parameters.

3.3. Comparison: Random versus Sequential Receptor Binding. In this section we compare time to entry $T$ between the two cases of random and sequential receptor binding. In particular, in Figure 10 we plot the ratio of the average time to entry $T_{\text {rand }} / T_{\text {seq }}$ for $N=4$ and $N=8$ as a function of $q, \mu_{0}$, and $D$. We consider the case where degradation only acts at the $n$ $=0$ binding state. The following analytical expression can be written for the dimensionless mean entry time when the diffusion constant $D \rightarrow \infty$ and the degradation $\mu_{0}=0:{ }^{42}$

$$
\begin{aligned}
T=\sum_{n=0}^{N-1} \frac{1}{(N-n)^{\alpha} c_{\infty}}\left(1+\sum_{j=0}^{n-1} \prod_{i=j}^{n-1} \frac{q(i+1)^{\alpha}}{(N-i)^{\alpha} c_{\infty}}\right)+ \\
\frac{1}{\kappa_{\text {in }}}\left(1+\sum_{j=0}^{n-1} \prod_{i=j}^{n-1} \frac{q(i+1)^{\alpha}}{(N-i)^{\alpha} c_{\infty}}\right)
\end{aligned}
$$

Here the choice of random $\alpha=1$ or sequential $\alpha=0$ binding is expressed through the forms used for $p_{n}$ and $q_{n}$ as given in eqs 6 and 7. Equation 15 is derived from a linear Markov chain of binding and unbinding events and assumes a reflecting boundary condition at the zero-receptor state $n=0$, and an absorbing boundary at the fusion state accessed from $n=N$. Numerical calculations of mean entry time $T$ as $D \rightarrow \infty$ and $\mu_{0}$ $=0$, for both random and sequential receptor binding, match the analytical expression (data not shown).

Figure 10 shows a maximum in the mean entry time ratio $T_{\text {rand }} / T_{\text {seq }}$ as a function of $q$. This nonmonotonic behavior is due to the unbinding and binding rates balancing one another at intermediate receptor occupancy when random binding is assumed. ${ }^{43}$ For random binding $p_{n} c(r=1, t)=(N-n) c(r=$ $1, t)$ and $q_{n}=n q$, both carrying an explicit $n$ dependence. Varying $q$ may then lead to $n^{*}$ values where $p_{n^{*}} c(r=1, t) \simeq$ $q_{n^{*}}$, forcing the system to spend more time about $n \simeq n^{*}$. Fluctuations about $n^{*}$ give rise to a kinetic trap, such that $p_{n} c(r$ $=1, t)>q_{n}$ for $n<n^{*}$ and $p_{n} c(r=1, t)<q_{n}$ for $n>n^{*}$, with a vanishing net drift across the occupation states close to $n^{*} .43$ Kinetic traps do not arise in the sequential case, since neither attachment nor detachment rates bear explicit $n$ dependencies.

We expect the effects of the kinetic trap to be enhanced for $n^{*} \sim N / 2$ and for larger $N$, when fluctuations do not allow for an easy escape from the trap and when the fusion or reflecting state at $n=N$ and $n=0$, respectively, are far. Furthermore, even if the trap is escaped, as $n$ approaches the fusion competent state $N$ the binding and unbinding rates will strongly push the system back toward it since $q_{n}=n q$ will be much larger than $(N-n) c(r=1, t)$. Indeed, in Figure 10, the peak occurs when the unbinding rate approaches $q \simeq c_{\infty}$ and rises dramatically for larger $N$. Upon lowering or raising $q, T_{\text {rand }}$ decreases, since the balancing no longer occurs for $n^{*} \simeq N / 2$ and it is easier to escape the kinetic trap, or reach either the fusion state or the reflecting boundary. As evident from Figure 10, only at very low values of $q$ does the virus enter the cell more quickly when random receptor binding is employed and $T_{\text {rand }} / T_{\text {seq }}<1$. In this limit, unbinding is extremely slow for both the random and the sequential models; however, the larger effective random binding rates at low receptor occupancy imply smaller mean entry times $T_{\text {rand. }}$.

Finally, we note that, at fixed values of $q, T_{\text {rand }} / T_{\text {seq }}$ may have a maximum as a function of $\mu_{0}$. The maximum in $\mu_{0}$ can be understood as follows. Although both $T_{\text {rand }}$ and $T_{\text {seq }}$ decrease with increasing $\mu_{0}$, due to their conditional nature, $T_{\text {rand }}$ reaches a plateau around $\mu_{0} \simeq N c_{\infty}$, while $T_{\text {seq }}$ does so around $\mu_{0} \simeq c_{\infty}$. For small $\mu_{0} \ll c_{\infty}$, the ratio $T_{\text {rand }} / T_{\text {seq }}$ increases for increasing $\mu_{0}$ since $T_{\text {seq }}$ decreases more than $T_{\text {rand }}$ in this regime. In the intermediate window $c_{\infty} \leq \mu_{0} \leq N c_{\infty}$, where $T_{\text {seq }}$ has reached its plateau and $T_{\text {rand }}$ is still decreasing, the ratio $T_{\text {rand }} / T_{\text {seq }}$ decreases as $\mu_{0}$ is increased. Finally, for large $\mu_{0} \gg N c_{\infty}$, both $T_{\text {rand }}$ and $T_{\text {seq }}$ have both reached their plateaus, leading to a constant ratio $T_{\text {rand }} / T_{\text {seq. }}$.

\section{Summary and Conclusions}

In this paper, we have developed a kinetic model for multistep viral entry by including viral degradation, receptor diffusion, and multiple binding of surface cellular receptors to viral glycoprotein ligand spikes. We studied the competition between factors that inhibit and promote fusion, by considering two alternate mechanisms driving the receptor binding process - random and sequential. We also considered two different ways for degradation to take place, uniformly across the multistep binding process that leads to fusion, or only at the unbound state $n=0$.

Our study reveals that most qualitative features of the viral entry process are not dependent on the chosen receptor binding model (random versus sequential) especially in the regime of kinetic parameters that are physically relevant, including moderate receptor concentrations as reported in the literature ${ }^{32}$ and a fairly low receptor unbinding rate. ${ }^{41}$ More important are the physical properties of the cellular system such as the receptor diffusion coefficient, receptor concentration, and the magnitude and acting mechanism of the degradation rate. These are all independent of viral properties 
and may perhaps help explain why certain cell types are more susceptible to HIV-1 entry. Carefully understanding how specific mechanisms (diffusion, degradation, attachment/ detachment) affect the overall entry process can have important implications for the development of antiretroviral therapies. As previously described, it has been recently shown ${ }^{35}$ that treating HIV-1 viral surfaces with the sphingomyelinase enzyme decreases the surface mobility of viral CD4 receptors, after the first virus - cell contact is established. Other recent studies show that HIV-1 fusion can be inhibited via drugs that specifically reduce CCR5 density levels, and that lower concentrations CCR5 result in lower fusion events. $^{34}$ Our work may serve to give these and similar experiments a quantitative framework to better understand how receptor diffusivity inhibits the fusion process.

Our model can be extended to study the effects of multiple receptor species on the entry process. For instance, HIV-1 is known to use both the CD4 receptor and the CCR5 or CXCR5 coreceptors, and the order of binding may not be random. Since the binding of the CD4 receptor causes the conformational change that allows for the subsequent binding of coreceptors, cellular concentrations and diffusion constants for different receptor/coreceptor species are not equivalent and ordering, competition, and cooperative effects may be important. ${ }^{4,27,31,33,34}$ All these features can be incorporated into an extended version of the model presented in this paper.

Acknowledgment. The authors of this work were supported by the National Science Foundation through grants DMS0719462 (MD) and DMS-0349195 (T.C.) and by the National Institutes of Health through grant K25AI058672 (T.C.).

\section{References and Notes}

(1) Dimitrov, D. S. Virus Entry: Molecular mechanisms and biomedical applications. Nat. Rev. 2004, 2, 109-122.

(2) Lamb, R. A. Paramyxovirus Fusion: A Hypothesis for Changes. Virology 1993, 197, 1-11.

(3) Doms, R. W. Beyond Receptor Exression: The Influence of Receptor Conformation, Density, and Affinity in HIV-1 Infection. Virology 2000, 276, 229-237.

(4) Kuhmann, S. E.; Platt, E. J.; Kozak, S. L.; Kabat, D. Cooperation of Multiple CCR5 Coreceptors Is Required for Infections by Human Immunodeficiency Virus Type 1. J. Virol. 2000, 74, 7005-7015.

(5) Yang, X.; Kurteva, S.; Ren, X.; Lee, S.; Sodroski, J. Stoichiometry of Envelope Glycoprotein Trimers in the Entry of Human Immunodeficiency Virus Type 1. J. Virol. 2005, 79, 12132-12147.

(6) Yang, X.; Kurteva, S.; Ren, X.; Lee, S.; Sodroski, J. Subunit Stoichiometry of Human Immunodeficiency Virus Type 1 Envelope Glycoprotein Trimers during Virus Entry into Host Cells. J. Virol. 2006, $80,4388-4395$.

(7) Yang, C.; Yang, Q.; Compans, R. W. Coreceptor-Dependent Inhibition of the Cell Fusion Activity of Simian Immunodeficiency Virus Env Proteins. J. Virol. 2000, 74, 6217-6222.

(8) Ragheb, J. A.; Anderson, W. F. pH-Independent Murine Leukemia Virus Ecotropic Envelope-Mediated Cell Fusion: Implications for the ROle of the R Peptide and p12E TM in Viral Entry. J. Virol. 1994, 68, 32203231.

(9) Urban, S. New Insights into Hepatitis B and Hepatitis Delta Virus Entry. Future Virol. 2008, 3, 253-264.

(10) Campadelli-Fiume, G.; Amasio, M.; Avitabile, E.; Cerretani, A.; Forghiere, C.; Gianni, T.; Menotti, L. The multipartite system that mediates entry of herpes simplex virus into the cell. Rev. Med. Virol. 2007, 17, $313-$ 326.

(11) Spear, P. G. Herpes simplex virus: receptors and ligands for cell entry. Cell. Microbiol. 2004, 6, 401-410.

(12) Bitzer, M.; Lauer, U.; Baumann, C.; Spiegel, M.; Gregor, M.; Neubert, W. J. Sendai Virus Efficiently Infects Cells vis the Asialoglycoprotein Receptor and Requires the Presence of Cleaved $F_{0}$ Precursor Proteins for This Alternative Route of Cell Entry. J. Virol. 1997, 71, 5481-5486.

(13) Russell, C. J.; Jardetzky, T. S.; Lamb, R. A. Membrane fusion machines of paramyxoviruses: capture of intermediates of fusion. $E M B O$ J. 2001, 20, 4024-4034.
(14) Wild, T. F.; Fayolle, J.; Beauverger, P.; Buckland, R. Measles Virus Fusion: Role of the Cysteine-Rich Region of the Fusion Glycoprotein. J. Virol. 1994, 68, 7546-7548.

(15) Negrete, O. A.; Levroney, E. L.; Aguilar, H. C.; Bertolotti-Ciarlet, A.; Nazarian, R.; Rajyar, S.; Lee, B. EphrinB2 is the entry receptor for Nipah virus, an emergent deadly paramyxovirus. Nature $\mathbf{2 0 0 5}, 436,401-$ 405.

(16) Negrete, O. A.; Wolf, M. C.; Aguilar, H. C.; Enterlein, S.; Wang, W.; Muhlberger, E.; Su, S. V.; Bertolotti-Ciarlet, A.; Flick, R.; Lee, B. Two key residues in ephrinB3 are critical for its use as an alternative receptor for Nipah virus. PLOS Pathogens 2006, 2, 78-86.

(17) Fallon, E. M.; Lauffenburger, D. A. Computational Model for Effects of Ligand/Receptor Binding Properties on Interleukin-2 Trafficking Dynamics and T Cell Proliferation Response. Biotechnol. Prog. 2000, 16, 905-916.

(18) Lieto, A. M.; Cush, R. C.; Thompson, N. L. Ligan-Receptor Kinetics Measured by Total Internal Reflection with Fluorescence Correlation Spectroscopy. Biophys. J. 2003, 85, 3294-3302.

(19) Bongrand, P. Ligand-receptor interactions. Rep. Prog. Phys. 1999, $62,921-968$

(20) Kuo, S. C.; Lauffenburger, D. A. Relationship between Receptor/ Ligand Binding Affinity and Adhesion Strength. Biophys. J. 1993, 65, 21912200

(21) Oda, K.; Matsuoka, Y.; Funahashi, A.; Kitano, H. A comprehensive pathway map of epidermal growth factor receptor signaling. Mol. Syst. Biol. 2005, 1, 2005-0010.

(22) Dushek, O.; Das, R.; Coombs, D. Analysis of membrane-localized binding kinetics with FRAP. Eur. Biophys. J. 2008, 37, 627-638.

(23) Chou, T. Stochastic Entry of Enveloped Viruses: Fusion versus Endocytosis. Biophys. J. 2007, 93, 1116-1123.

(24) Nowak, S. A.; Chou, T. Mechanisms of Receptor/CoreceptorMediated Entry of Enveloped Viruses. Biophys. J. 2009, 96, 2624-2636.

(25) van Effenterre, D.; Roux, D. Adhesion of colloids on a cell surface in competition for mobile receptors. Europhys. Lett. 2003, 64, 543-549.

(26) Chou, T.; D’Orsogna, M. R. Multistage Adsorption of Diffusing Macromolecules and Viruses. J. Chem. Phys. 2007, 127, 105101.

(27) Lee, B.; Sharron, M.; Montaner, L. J.; Weissman, D.; Doms, R. W. Quantification of CD4, CCR5, and CXCR4 levels on lymphocyte subsets, dendritic cells, and differentially conditioned monocyte-derived macrophages. Proc. Natl. Acad. Sci. 1999, 96, 5215-5220.

(28) Singer, I. I.; et al. CCR5, CXCR4, and CD4 are clustered and closely apposed on microvilli of human macrophages and T cells. J. Virol. 2001, 75, 3779-3790.

(29) Steffens, C. M.; Hope, T. J. Localization of CD4 and CCR5 in Living Cells. J. Virol. 2003, 77, 4985-4991.

(30) Baker, A.-M.; Sauli, A.; Gaibelet, G.; Lagane, B.; Mazères, S.; Fourage, M.; Bachelerie, F.; Salomè, L.; Lopez, A.; Dumas, F. CD4 Interacts Constitutively with Multiple CCR5 at the Plasma Membrane of Living Cells. J. Biol. Chem. 2007, 282, 35163-35168.

(31) Steffens, C. M.; Hope, T. J. Mobility of the Human Immunodeficiency Virus (HIV) Receptor CD4 and Coreceptor CCR5 in Living Cells: Implications for HIV Fusion and Entry Events. J. Virol. 2004, 78, 95739578.

(32) Platt, E. J.; Wehrly, K.; Kuhmann, S. E.; Chesebro, B.; Kabat, D. Effects of CCR5 and CD4 Cell Surface Concentration on Infections by Macrophagetropic Isolates of Human Immunodeficiency Virus Type 1. J. Virol. 1998, 72, 2855-2864.

(33) Reynes, J.; Portales, P.; Segondy, M.; Baillat, V.; André, P.; Réant, B.; Avinens, O.; Couderc, G.; Benkirane, M.; Clot, J.; Eliaou, J.-F.; Corbeau, P. $C D 4^{+} \mathrm{T}$ Cell Surface CCR5 Density as a Determining Factor of Virus Load in Persons Infected with Human Immunodeficiency VIrus Type 1. J. Infect. Dis. 2000, 181, 927-932.

(34) Heredia, A.; Gilliam, B.; DeVico, A.; Le, N.; Bamba, D.; Flinko, R.; Lewis, G.; Gallo, R. C.; Redfield, R. R. CCR5 density levels on primary CD4 T cells impact the replication and Enfuvirtide susceptibility of R5 HIV-1. AIDS 2007, 21, 1317-1322.

(35) Finnegan, C. M.; Rawat, S. S.; Cho, E. H.; Guiffre, D. L.; Lockett, S.; Merrill, A. H., Jr.; Blumenthal, R. Sphingomyelinase Restricts the Lateral Diffusion of CD4 and Inhibits Human Immunodeficiency Virus Fusion. J. Virol. 2007, 81, 5294-5304.

(36) Rawat, S. S.; Zimmerman, C.; Johnson, B. J.; Cho, E.; Lockett, S. J.; Blumenthal, R.; Puri, A. Restricted lateral mobility of plasma membrane CD4 impairs HIV-1 envelope glycoprotein mediated fusion. Mol. Membr. Biol. 2008, 25, 83-94.

(37) Seisenberger, G.; Ried, M. U.; Endress, T.; Büning, H.; Hallek, M.; Bräuchle, C. Real-Time Single-Molecule Imaging of the Infection Pathway of an Adeno-Associated Virus. Science 2001, 294, 1929-1932.

(38) Baker, T. S.; Olson, N. H.; Fuller, S. D. Adding the Third Dimension to VIrus Life Cycles: Three-Dimensional Reconstruction of Icosahedral Viruses from Cryo-Electron Micrographs. Microbiol. Mol. Biol. Rev. 1999, 63, 862-922. 
(39) Hildebrand, F. B. Finite-difference equations and simulations; Prentice-Hall: Englewood Cliffs, NJ, 1968.

(40) Butcher, J. C. Numerical methods for ordinary differential equations; John Wiley \& Sons: New York, 2003.

(41) Chang, M. I.; Panorchan, P.; Dobrowsky, T. M.; Tseng, Y.; Wirtz, D. Single-Molecule Analysis of Human Immunodeficiency Virus Type 1 gp120Receptor Interactions in Living Cells. J. Virol. 2005, 79, 14748-14755.
(42) Pury, P. A.; Cáceres, M. O. Mean first-passage and residence times of random walks on asymmetric disordered chains. J. Phys. A: Math. Gen. 2003, 36, 2695-2706.

(43) Chou, T.; D’Orsogna, M. R. First Passage and Cooperativity of Queuing Kinetics. Phys. Rev. Lett. 2005, 95, 170603.

JP1080725 